\title{
Use of apical suction to facilitate extra-anatomic bypass for recurrent coarctation: a case report Manoj Kuduvalli*1,2, Colin Monaghan ${ }^{1}$ and Brian M Fabri ${ }^{1}$
}

Address: ${ }^{1}$ Department of Cardiothoracic Surgery, The Cardiothoracic Centre, Liverpool, UK and ${ }^{2}$ Specialist Registrar, Cardiothoracic Surgery, The Cardiothoracic Centre NHS Trust, Thomas Drive, Liverpool, L14 3PE, UK

Email: Manoj Kuduvalli* - manoj.kudavalli@ctc.nhs.uk; Colin Monaghan - colin.monaghan@ctc.nhs.uk; Brian M Fabri - Brian.Fabri@ctc.nhs.uk

* Corresponding author

Published: 28 March 2006

Journal of Cardiothoracic Surgery 2006, I:9 doi:10.1186/1749-8090-1-9
Received: 26 January 2006

Accepted: 28 March 2006

This article is available from: http://www.cardiothoracicsurgery.org/content/I/I/9

(c) 2006 Kuduvalli et al; licensee BioMed Central Ltd.

This is an Open Access article distributed under the terms of the Creative Commons Attribution License (http://creativecommons.org/licenses/by/2.0), which permits unrestricted use, distribution, and reproduction in any medium, provided the original work is properly cited.

\begin{abstract}
The use of apical suction devices has been well described for maintaining satisfactory haemodynamics during off-pump surgical coronary revascularization. Its expanded use has been described in a few other situations. We describe here a case of recurrent coarctation where an extra-anatomic ascending to descending thoracic aorta bypass graft was constructed using cardiopulmonary bypass without arresting the heart, and access and exposure were facilitated by the use of an apical suction device.
\end{abstract}

\section{Case report}

A 49 year old gentleman presented to cardiology with lower limb claudication pain and breathlessness of three years duration. Clinical examination revealed upper limb hypertension, with similar blood pressures in both arms $(180 / 100 \mathrm{~mm} \mathrm{Hg})$. His past history included repair of coarctation of aorta about 30 years ago. The medical records and operative details from the previous operation were unavailable. The operation had been performed through a left thoracotomy. An MRI scan revealed a $2 \mathrm{~cm}$ long narrowing of the aorta just distal to the origin of an aberrant right subclavian artery, which was the last of four branches from the aortic arch (Fig. 1). The origins of the arch vessels did not show any sign of narrowing. The aortic root and ascending aorta were $3.5 \mathrm{~cm}$ in diameter, and the arch was of normal calibre. The diameter in the region of the stenosis was $1.4 \mathrm{~cm}$ with an additional web-like stenotic lesion at the distal end of the stenotic segment. There was evidence of calcification, possibly of an interposition tube graft which had been used at the time of the first operation. The descending thoracic aorta was of normal calibre.

In view of his symptomatic status, a re-intervention was considered appropriate. In view of his previous surgery, and especially the fact that the area of re-coarctation appeared to be calcified, it was decided to approach the aorta via a median sternotomy and construct an extra-anatomic ascending to descending thoracic aorta bypass graft. Cardiopulmonary bypass would be necessary to lift the heart out of the way to gain access to the descending thoracic aorta just above the diaphragm. We planned to use an apical suction device to keep the empty beating heart elevated.

The sternotomy was completed uneventfully. The pericardial cavity was obliterated with dense adhesions. This was rather surprising since we had anticipated that the previous procedure would have been extra-pericardial. However, further dissection revealed a large hole in the pericardial sac with the left lung directly adherent to the 


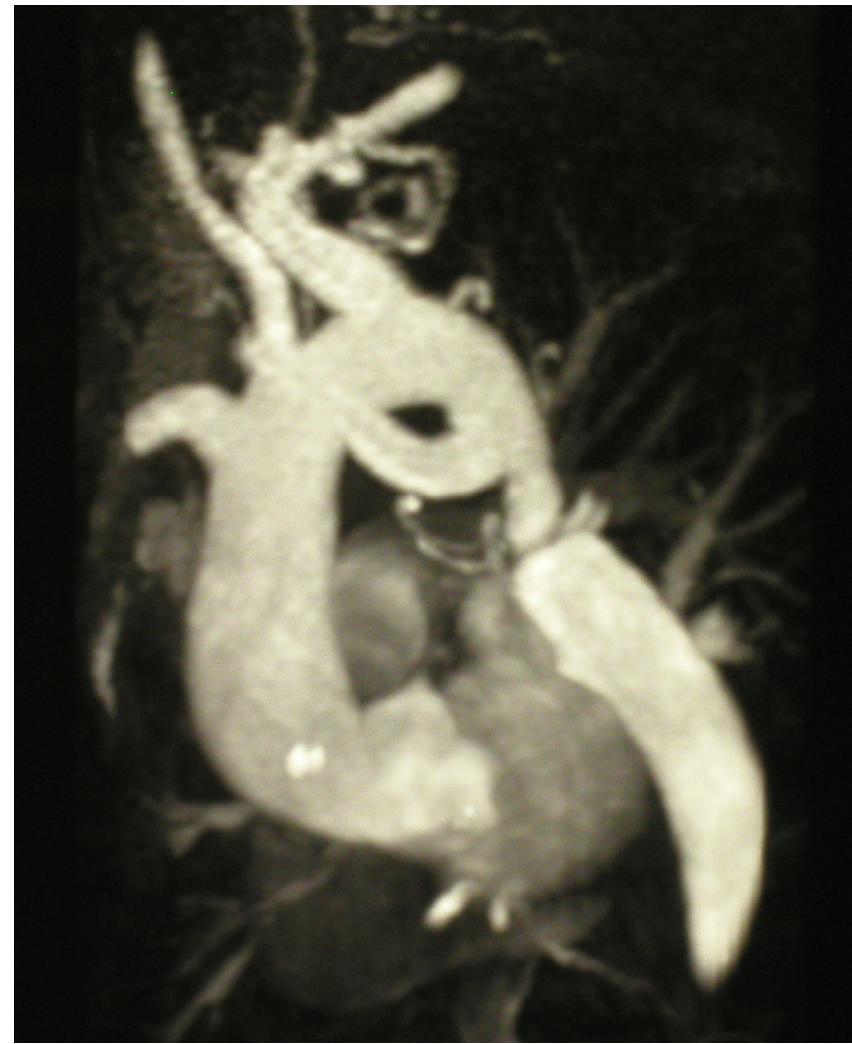

Figure I

MRI scan showing the re-coarctation.

heart. The adhesions were released, some of them after establishing cardiopulmonary bypass using ascending aortic cannulation for inflow and bicaval cannulation (to maintain adequate venous drainage even after lifting up

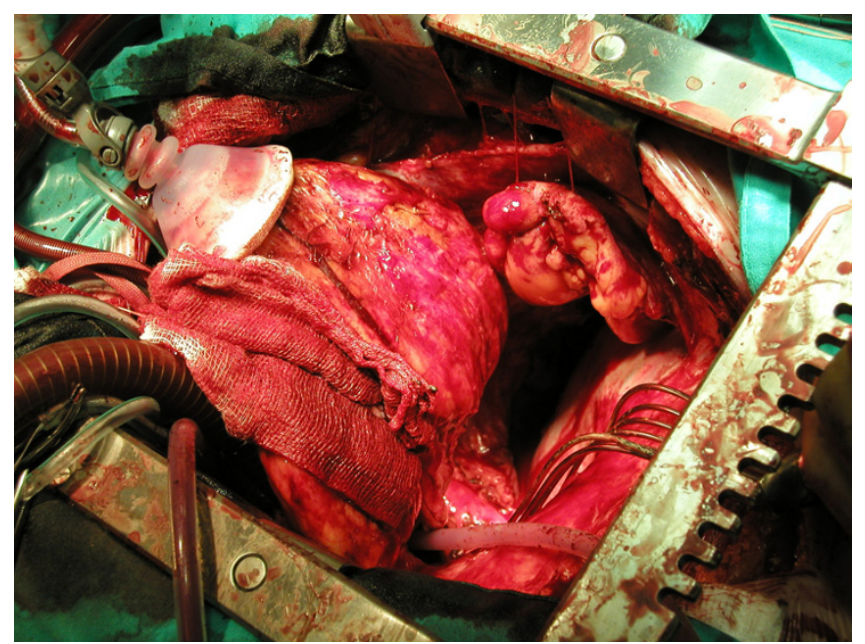

Figure 2

Exposure of the descending thoracic aorta with the apical suction device retracting the heart. the heart) for venous outflow. Once the apex and the posterior surface of the heart were free of adhesions, an apical suction device (URCHIN ${ }^{\mathrm{TM}}$ Heart Positioner, Medtronic Inc., Minneapolis MN55432-5604 USA) was placed in position and the beating heart was lifted superiorly. This allowed further dissection in the posterior pericardium and allowing freeing up of adhesions between the left lung and the descending thoracic aorta, and allowed visualization of and access to the descending thoracic aorta just above the diaphragm in spite of a deep thoracic cavity (Fig. 2). Proximal and distal cross clamps were applied isolating a $4 \mathrm{~cm}$ length of aorta. A longitudinal incision was made in this segment and an $18 \mathrm{~mm}$ Haemashield Platinum $^{\mathrm{TM}}$ Woven Double Velour Vascular tube graft (Boston Scientific Corporation, Natick, MA 01760-1537) was anastomosed in an end to side manner using continuous 3-0 polypropylene sutures. The clamps were released, the distal one first, the aorta was de-aired and the anastomosis was checked. The graft was then routed to the right of the inferior vena cava and brought up alongside the right atrium to the ascending aorta. The apical suction device was released and the heart was replaced in the pericardial sac. The length of the tube graft was estimated after filling up the heart. A side biting clamp was applied to the ascending aorta and the proximal anastomosis of the tube graft was constructed to a longitudinal arteriotomy using 3-0 polypropylene sutures. The clamp was released, the graft was de-aired, and the anastomosis checked. The patient was weaned off cardiopulmonary bypass with no inotropic support. Haemostasis was ensured and the chest was closed in the routine manner leaving two drains in the left pleural space, one drain in the pericardial sac and one in the mediastinum.

The patient was extubated eight hours after arrival in the intensive care unit. His drains were removed the next morning. He made an uneventful recovery thereafter except for needing some respiratory support with noninvasive continuous positive airway pressure for treating basal atelectasis. A CT scan was done prior to his discharge from hospital on the tenth postoperative day. Figure 3 shows an oblique 3-D reconstructed view from the CT scan demonstrating the locations of the proximal and distal anastomoses, and the lie of the graft. The patient was reviewed in outpatients six weeks after his discharge. His claudication pain had disappeared completely. His upper limb blood pressure was $120 / 60 \mathrm{~mm} \mathrm{Hg}$ on a reduced amount of medication.

\section{Comment}

The use of apical suction devices for cardiac positioning in off pump coronary artery surgery, and its ability to maintain good beating heart dynamics has been described $[1,2]$. Apart from being used in off-pump coronary artery bypass grafting, the apical suction device has been 


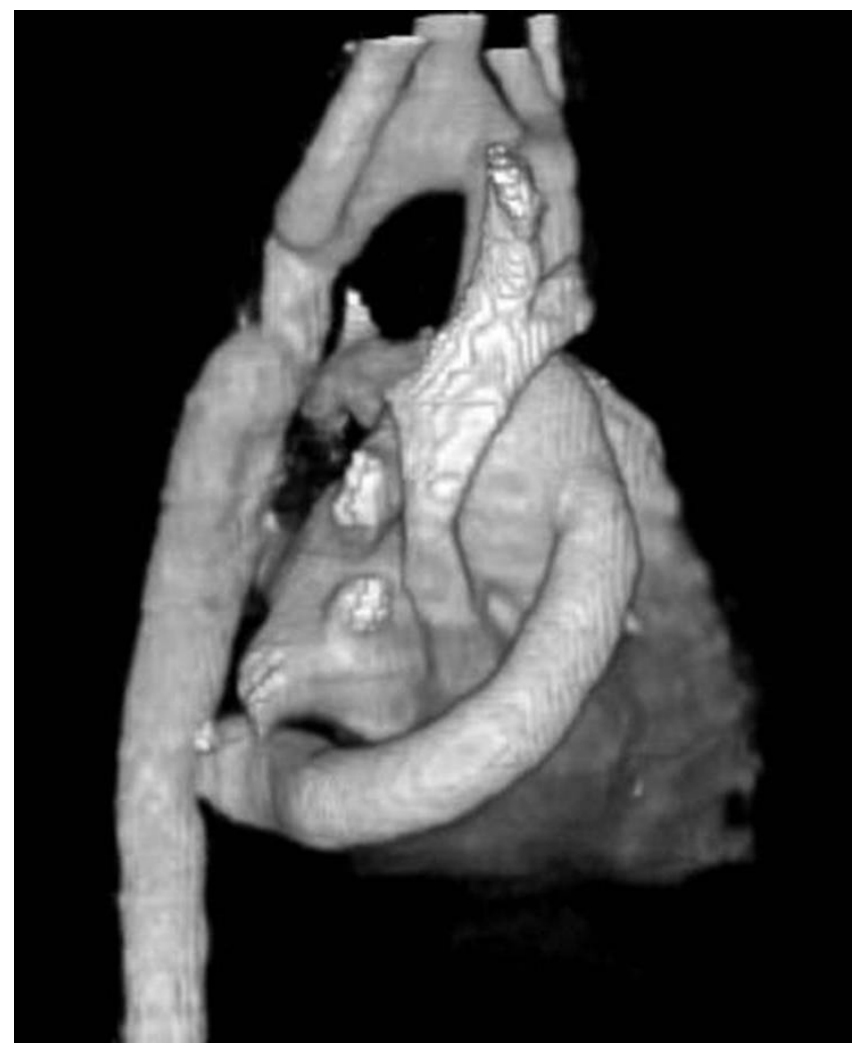

Figure 3

3-D reconstruction of postoperative CT scan showing the extra-anatomic bypass graft.

described to have expanded uses in a variety of scenarios. It has been described for use in pericardectomies, during lysis of adhesions in redo coronary surgery, for securing epicardial haemostasis in penetrating cardiac trauma, for securing haemostasis during re-exploration after cardiac surgery and for facilitating epicardial microwave ablation [3].

Its use has also been described in a case of recurrent coarctation in which an ascending-to-abdominal aorta bypass graft was successfully facilitated by the use of an apical suction device [4]. The operation was done without the use of cardiopulmonary bypass.

In the case described in our report, the use of cardiopulmonary bypass was necessary because the heart would probably not have tolerated the amount of elevation which would have been required to expose the descending thoracic aorta just above the diaphragm, which was our target area for constructing the anastomosis. However, our rationale for use of the apical suction device on the empty beating heart was: 1) to avoid cross clamping the heart for a prolonged period of time for an extra-cardiac operation 2) to make the elevation and retraction of the empty beating heart technically easier, and less traumatic on the epicardium and myocardium compared to retracting and elevating with the use of the assistant's hand. We could accomplish both these objectives safely and successfully, with adequate exposure to clamp the descending thoracic aorta and perform the anastomosis.

This report describes another expanded use for the apical suction device.

\section{References}

I. Chang WI, Kim KB, Kim JH, Ham BM, Kim YL: Haemodynamic changes during posterior vessel off-pump coronary artery bypass:comparison between deep pericardial sutures and vacuum-assisted apical suction device. Ann Thorac Surg 2004, 78(6):2057-62.

2. Sepic J, Wee JO, Soltesz EG, Hsin MK, Cohn LH, Laurence RG, Aklog $\mathrm{L}$ : Cardiac positioning using an apical suction device maintains beating heart hemodynamics. Heart Surg Forum 2002 5(3):279-84.

3. Athanasiou T, Kumar P, Al-Ruzzeh S, Aziz O, Charitou A, Hart J, Glenville B, Casula R: Expanded use of suction and stabilization devices in cardiothoracic surgery. Ann Thorac Surg 2003, 76(4): I I 26-30

4. Athanasiou T, Aziz T, Aziz O, Cheshire N, Stanbridge RD: Apical suction device facilitating off-pump bypass for recurrent coarctation: case report. Heart Surg Forum 2004, 7(2):EI77-8.
Publish with BioMed Central and every scientist can read your work free of charge

"BioMed Central will be the most significant development for disseminating the results of biomedical research in our lifetime. " Sir Paul Nurse, Cancer Research UK

Your research papers will be:

- available free of charge to the entire biomedical community

- peer reviewed and published immediately upon acceptance

- cited in PubMed and archived on PubMed Central

- yours - you keep the copyright
BioMedcentral 